\title{
Perception of relative pitch with different references: Some absolute-pitch listeners can't tell musical interval names
}

\author{
KEN'ICHI MIYAZAKI \\ Niigata University, Niigata, Japan
}

\begin{abstract}
Two experiments were conducted to examine the effect of absolute-pitch possession on relativepitch processing. Listeners attempted to identify melodic intervals ranging from a semitone to an octave with different reference tones. Listeners with absolute pitch showed declined performance when the reference was out-of-tune $\mathrm{C}$, out-of-tune $\mathrm{E}$, or $\mathrm{F} \#$, relative to when the reference was $\mathrm{C}$. In contrast, listeners who had no absolute pitch maintained relatively high performance in all reference conditions. These results suggest that absolute-pitch listeners are weak in relative-pitch processing and show a tendency to rely on absolute pitch in relative-pitch tasks.
\end{abstract}

One of the most important areas in our auditory experience where pitch is used in a highly elaborated manner is music. In music of the tonal harmonic idiom, pitch, in association with time, provides a tonal space in which melodic and harmonic events occur in a hierarchical manner. Musical tones have different degrees of stability depending on the established tonal context (Lerdahl, 1988; Piston, 1987). For example, the first degree note of the diatonic scale (the tonic) is the most stable, followed by the fifth note (the dominant), and the nondiatonic notes are the least stable. Several experiments have provided evidence for the psychological validity of tonal hierarchies (Krumhansl, 1990).

In music, absolute pitch in itself does not convey musically important meanings, but musical tones make sense only when they are located in a tonal context given by a particular pitch set. A given pitch can represent different musical meanings if it is placed in different tonal contexts (e.g., the $\mathrm{C}$ note functions as the tonic in the $\mathrm{C}$ major key context and as the dominant in the $\mathrm{F}$ major context), or conversely, different pitches can be perceived as having the same musical character if they are located at the same position in different tonal contexts (e.g., the $G$ and $D$ notes function as the dominant in the $\mathrm{C}$ major and $\mathrm{G}$ major, respectively). Moreover, melodic sequences can be transposed to any pitch position without destroying their identity. This phenomenon of invariance in musical pitch patterns has drawn considerable attention from psychologists in the Gestalt tradition. Attneave and Olson (1971) noted that pitch is a morphophoric, or form-bearing, prop-

This research was supported by a Grant-in-Aid for Scientific Research (No. 04610043) from the Japanese Ministry of Education, Science and Culture, to the author. Correspondence should be addressed to Ken'ichi Miyazaki, Department of Psychology, Faculty of Humanities, Niigata University, Ikarashi 2-no-cho, Niigata 950-21, Japan (e-mail: miyazaki@geb.ge.niigata-u.ac.jp). erty and could be regarded as a medium in which tonal patterns occur.

People who have good absolute pitch have been admired as highly talented musicians. Indeed, absolute pitch may be quite useful in musical practice, particularly when transcribing a heard musical passage into a musical notation or singing a complicated melody at first sight. However, it could be argued that the ability of absolute pitch is irrelevant to music when considering the ultimate importance of pitch relationship in music. In a sense, absolute pitch may be a sort of primitive ability. It is suggestive in this respect that some animals (for example, songbirds) show strong constraints on absolute pitch in identifying pitch patterns (Hulse, 1989). There is also some evidence suggesting that younger children are relatively sensitive to absolute-pitch features in singing well-memorized songs, but the balance shifts to the relative-pitch features with age (Sergeant \& Roche, 1973; Takeuchi \& Hulse, 1993, for review).

Absolute pitch and relative pitch are quite different modes of pitch processing and may be incompatible in some cases. A report of successful training of absolute pitch in children (Oura \& Eguchi, 1982) showed that absolute pitch is most efficiently acquired before the age of 7 , after which relative pitch develops. This shift suggests that the development of relative pitch may make it difficult to acquire absolute pitch (Hulse, Takeuchi, \& Braaten, 1992). There may also be a reverse effect-that is, absolute pitch once acquired could interfere with the full development of relative pitch, because children who acquired precise absolute pitch early in childhood would persist in using absolute pitch in musical activities and would have no motivation to learn relative pitch. Some music students who have precise absolute pitch say that they are relatively weak in certain relative-pitch tasks, such as identifying musical intervals in different tonal contexts or singing a given notated melody in different keys. Furthermore, musicians with precise absolute pitch often say that they feel uncomfortable when listening to music played by instruments tuned 
to a slightly shifted tuning system, as in the case of instruments tuned with the historical pitch standard that is slightly lower than the current one.

It is therefore interesting to examine whether people with absolute pitch really have any difficulty in recognizing pitch relations in different reference conditions. This question was examined in a previous experiment (Miyazaki, 1992), in which listeners with absolute pitch attempted to identify melodic intervals of various sizes in terms of traditional musical interval classes. The reference tone (the first tone of the interval) was either in-tune $\mathrm{C}$ or out-of-tune $C$. The subjects showed significantly lower performance and longer response time for the out-of-tune reference than for the in-tune reference. However, this experiment included only one condition of the out-of-tune reference (quarter tone higher than the standard C). It is not clear how much deviation is effective in reducing performance.

Experiment 1 of the present investigation was carried out to examine the effect of pitch deviation on the accuracy of relative-pitch judgment using four reference tones with various amounts of deviation from the standard pitch. In Experiment 2, test intervals were presented with one of three references having clearly different pitch classes $(C$, $\mathrm{F} \#$, and a quarter-tone-lower $\mathrm{E}$ ). If absolute-pitch listeners tend to stick to absolute pitch even in perceiving musical pitch relations, they should have some difficulty when the reference is not $C$. Because such listeners are not accustomed to perceive pitch relations with reference to unstable notes other than $C$, they would presumably have to use complicated strategies based on absolute pitch, such as identifying two pitches separately and then counting the number of semitone steps between the two tones.

\section{EXPERIMENT 1}

The purpose of Experiment 1 was to investigate how accurately absolute-pitch possessors could categorize pitch relations between two successive tones when the pitch of the reference tone varied. The pitch intervals between the stimulus tones ranged from 50 cents to 1,230 cents ( 1 cent $=1 / 100$ of a semitone in equal temperament), thus including a number of intervals mistuned from the conventional equal temperament. The first tone of the interval (the reference) was in-tune $\mathrm{C}$ or out-of-tune $\mathrm{C}$. The primary concern of the experiment was to examine to what degree identification performance would deteriorate with out-oftune references.

\section{Method}

Subjects. The subjects were 8 students ( 3 males and 5 females) in a music education course at Niigata University. They were selected according to the criterion of more than $90 \%$ correct responses in a preliminary absolute-pitch test using 60 tones over five octaves. All had received musical instruction in playing the piano when they were 3 to 5 years old. They were paid for their services.

Stimulus and Apparatus. The stimuli presented were musical intervals of various sizes formed by pairs of successive tones. Fundamental frequencies of the tones were determined according to equal temperament with reference to the standard pitch of $440 \mathrm{~Hz}$ as
$\mathrm{A}_{4}$. The first tone of the pair was a reference tone for judging the interval. There were four different contexts established by four reference tones of slightly different frequencies: one in-tune $C_{4}(261.6$ $\mathrm{Hz})$ and three out-of-tune references, 16,30 , and 50 cents higher than $\mathrm{C}_{4}(264.1,266.2$, and $269.3 \mathrm{~Hz}$, respectively). The second tone was higher than the reference by an interval that ranged from a minor second ( 100 cents) to an octave $(1,200$ cents), plus deviations of $-50,-30,-16,0,16$, or 30 cents. Thus, minor seconds ranged from 50 to 130 cents, major seconds from 150 to 230 cents, and so on, for a total of 72 different intervals. Each stimulus tone was a synthesized piano-like tone with an amplitude envelope of a fast attack and a slow decay and a duration of about $1.2 \mathrm{sec}$. The time interval between the onsets of the two tones was $1.4 \mathrm{sec}$.

Stimuli were produced by a synthesizer (Yamaha DX-7), using its built-in piano-like timbre. After being amplified by a power amplifier (Pioneer M-30), the stimuli were presented to the subjects through a loudspeaker (Yamaha NS-1000M) in a quiet room at a comfortable level. A computer (NEC PC-980IVM2) assisted by a Musical Instrument Digital Interface (MIDI) processing unit (Roland MPU-401) was used in controlling the experimental equipment and registering the subjects' responses.

Procedure. In each trial, the subjects attempted to identify the pitch of the target tone with its relative pitch name (for example, "mi" or "sol" in the movable-do system) or its conventional musical interval name in relation to the reference tone. The subjects were instructed to establish a tonal context for judging relative pitch with the first tone as "do" in the movable-do naming system; specifically, they were encouraged to have a mental representation of the major diatonic scale with the reference as a tonal center.

The subjects made responses by pressing one of the keys on a computer keyboard. There were 12 main response categories from the minor second to the octave. In addition, there wcre two subcategories ("lower" and "higher") for each of the 12 main categories. The main categories and the subcategories were assigned to different key arrays on the computer keyboard. The subjects had the option of using these subcategories when they perceived the interval as mistuned. On each response key, a label indicated a corresponding response category name (both a relative pitch name and a musical interval name). Experimental sessions were self-paced: when the subjects were ready for the next trial, they pressed a space bar, which triggered the stimulus for the next trial with a $1-$ sec delay.

Seventy-two trials constituted one trial block, in which each of 72 different intervals appeared once with one type of reference. Four blocks of trials with different references each made up a 1-day session. It took about 30 min to complete a block, and there were sufficient rest periods between the blocks. In total, four experimental sessions were conducted over four separate days; thus, there were four trials for each stimulus with each reference. The order of trial blocks was counterbalanced among sessions and subjects.

\section{Results and Discussion}

The overall response distributions pooled over the 8 subjects are displayed as a set of identification functions in Figure 1 for the two extreme conditions with the in-tune $\mathrm{C}$ and the 50 -cent-higher $\mathrm{C}$ references. In this figure, the percentage of responses identifying each interval category is represented as a function of interval size; for the sake of simplification, the three subcategories ("smaller," "intune," and "larger") for each musical interval were combined into a response category for that interval.

It is evident that musical intervals were categorized quite consistently, resulting in a set of identification functions similar to those previously reported by Burns and Ward (1978), Siegel and Siegel (1977), and Rakowski (1990). More important for the present purpose, for some 


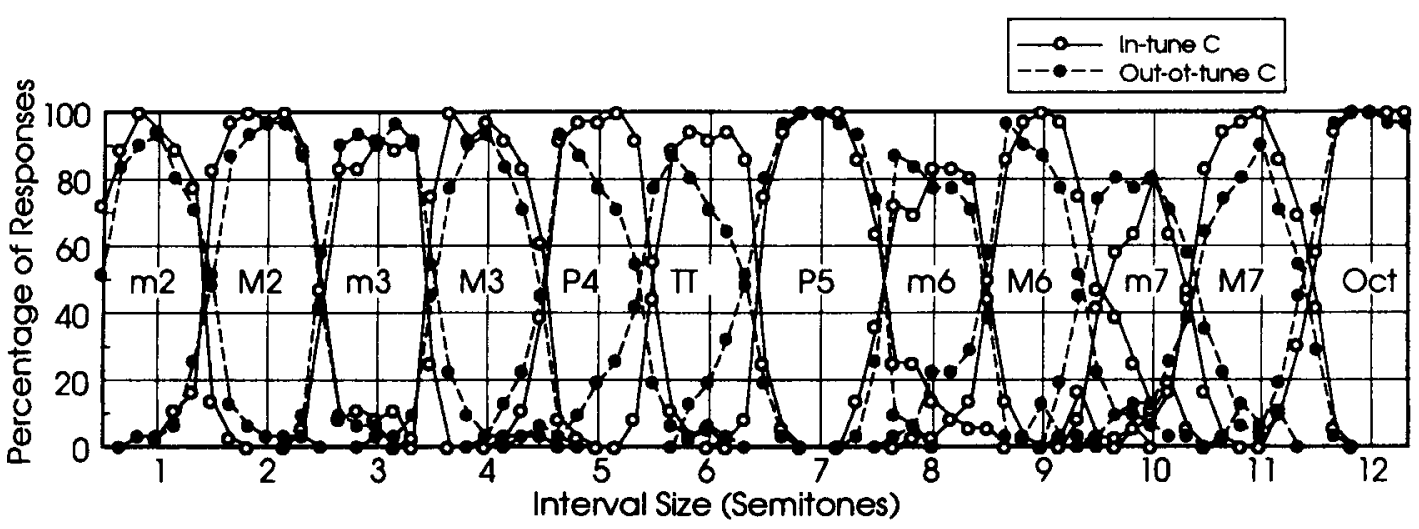

Figure 1. Percentage of identification responses pooled over 8 absolute-pitch listeners in conditions with in-tune $C$ reference (open circles) and 50-cent-higher $\mathrm{C}$ reference (filled circles) as a function of musical interval size. The label at each function denotes the musical interval name in an abbreviated form: $P 5=$ the perfect 5 th, $T$ T $=$ the tritone, Oct $=$ the octave, $\mathrm{m} 2$ = the minor $2 \mathrm{nd}, \mathrm{M} 2$ = the major $2 \mathrm{nd}$, and so on.

larger intervals (for example, perfect 4th, tritone, major 6 th, and minor 7 th), the distributions in the deviated reference condition are slightly skewed toward the direction of smaller intervals, whereas those with the in-tune $\mathrm{C}$ reference are nearly symmetric in shape around the points of the corresponding interval value. Such skewness may reflect a strategy some absolute-pitch subjects used identifying larger intervals, which are relatively difficult to identify by using relative pitch. The strategy is to judge the deviated $\mathrm{C}$ as a somewhat acceptable $\mathrm{C}$ and to judge a slightly smaller interval as acceptable more often than an exact interval. For example, if the 50-cent-higher $\mathrm{C}$ reference was perceived as acceptable $\mathrm{C}$, a target tone that was higher by 600 cents from the in-tune $C$ would be perceived as an acceptable tritone in spite of the fact that the interval actually presented was 550 cents. If this strategy was used, it was not used by all subjects: the amount of peak shift was less than expected from the 50-cent deviation. Examining the individual results separately, there were 3 subjects out of 8 who showed a noticeable difference in response pattern between the two reference conditions.

The shift of the response pattern was observed only for certain larger intervals; for small intervals (minor 2 nd through major 3rd) and diatonically important larger intervals (perfect 5 th and octave), the distributions are nearly the same in the in-tune $\mathrm{C}$ and out-of-tune $\mathrm{C}$ reference conditions. These results indicate that the subjects did not always use absolute pitch but might have used absolute pitch and relative pitch flexibly, depending on the difficulty in the task. Smaller intervals and diatonically important ones are assumed to be easier to perceive as pitch relationships, since they are melodically or harmonically important and consequently well learned.

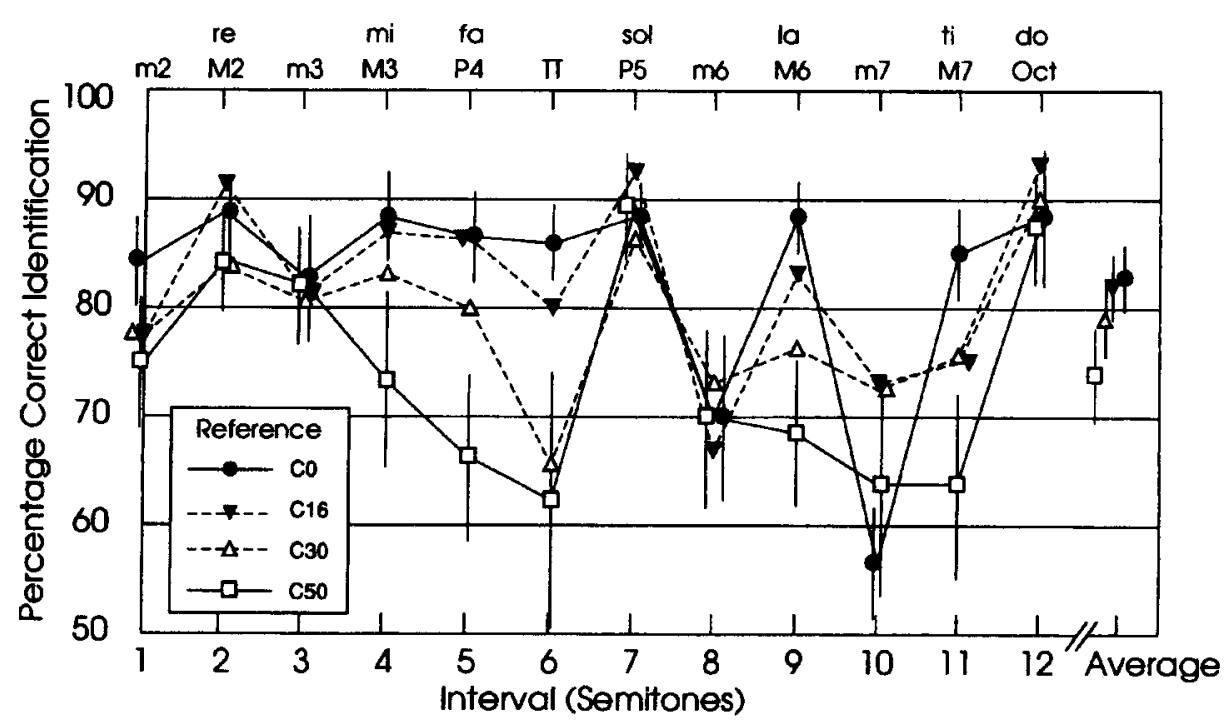

Figure 2. Average percentage of correct identification of musical intervals as a function of musical interval size in different reference conditions. Error bars on data for $\mathrm{C} 0$ and $\mathrm{C} 50$ reference conditions and overall averages show $\pm 1 S E$. 
To compare the performance more simply, the percentage of correct identification was calculated for each of 12 chromatic interval categories, including five adjacent original stimuli with -30 - to +30 -cent deviations but excluding intervals with 50 -cent deviations. Intervals that deviated by 16 or 30 cents were counted as correctly identified if they were recognized either as exactly the nearest nominal interval or as deviating in the corresponding direction. For example, the 216-cent interval was counted as correctly identified when the response was either "exact" or "wider major 2nd." The responses to the physically exact intervals were counted as correct when they were either "smaller," "exact," or "wider" for those intervals. The result averaged over the 8 subjects is displayed in Figure 2.

Identification performance depends heavily on interval categories. An analysis of variance (ANOVA) with reference and interval as within-subject factors showed that the effect of interval was highly significant $[F(11,77)=3.22$, $p<.002]$. The differences in ease of identification among relative pitches appear to reflect the well-demonstrated tonal hierarchy found among notes in a tonal context: tonally important notes (do and sol) are most stable, followed by other diatonic scale notes, while nondiatonic notes are least stable (for example, Krumhansl \& Shepard, 1979). A multiple regression analysis was conducted to examine the extent to which the identification performance in each reference condition reflected tonal hierarchy (the probe tone ratings reported by Krumhansl \& Kessler, 1982) and interval size (the number of semitones between the reference and the target). The multiple regression coefficient for every reference condition was significant ( $p<.05$ for the $\mathrm{C} 0$ and $\mathrm{C} 50$ conditions; $p<.005$ for the $\mathrm{C} 16$ and $\mathrm{C} 30$ conditions). The contribution of the tonal hierarchy was greater than that of the interval size. Normalized regression coefficients (beta weights) for tonal hierarchy were $.68(p<.03)$ for $\mathrm{C} 0, .93(p<.001)$ for $\mathrm{C} 16, .91(p<.001)$ for $\mathrm{C} 30$, and $.72(p<.02)$ for $\mathrm{C} 50$, whereas those for interval size, ranging from -.28 to -.51 , were insignificant, except for C16 $(p<.01)$. Miyazaki (1989) found a similar profile and a related difference in accuracy/speed between white- and black-key notes for pitch identification with absolute-pitch possessors (see also, Takeuchi \& Hulse, 1991). It is possible, therefore, that the observed differences in performance among relative pitches may reflect this hierarchy among absolute pitches for the absolute-pitch subjects.

The significant effect of reference in the ANOVA $[F(3,21)=7.62, p<.002]$ arose because identification performance in the in-tune $\mathrm{C}$ context $(\mathrm{C} 0)$ was, in most cases, higher than in the most deviant context (C50). The performance in the two other deviant contexts ( $\mathrm{Cl} 6$ and $\mathrm{C} 30)$ tended to be intermediate between the two extremes, although there are some irregularities. Planned comparisons of overall performance levels among reference conditions suggested that the performance in C50 was significantly lower than any other contexts $(p<.03)$, whereas the differences between means in $\mathrm{C} 0, \mathrm{C} 16$, and $\mathrm{C} 30$ were not significant. These comparisons suggest that the relative-pitch identification of the absolute-pitch listeners be- gins to deteriorate when the reference deviates from the in-tune $\mathrm{C}$ by more than 30 cents. It should be added that the middle $\mathrm{C}$ is one of the most stable anchors for musicians (especially for keyboard players) and, therefore, the value of effective deviation may be somewhat different for other referent tones.

The interaction of reference $\times$ interval was also significant $[F(33,231)=1.72, p<.02]$, indicating that the amount of decline in performance in the out-of-tune condition depended on intervals. The performance in C50 was markedly lower than in $\mathrm{C} 0$ for intervals of $5,6,9$, and 11 semitones, whereas the performance was nearly the same among reference conditions for intervals of smaller size (e.g., less than 3 semitones) and of tonal importance (e.g., the perfect 5 th and octave).

Examining the individual results, 6 subjects out of 8 showed an overall tendency for the percentage of correct identification of relative pitch to decrease with the deviation of the referent tone. If the subjects did the task completely on the basis of relative pitch, the performance would have been at the same level regardless of the amount of deviation. The observed decline of performance suggests that the relative-pitch judgment of the absolute-pitch subjects was not completely relative but was to some extent influenced by their absolute pitch. It may be that the deviant reference tone sounded as if it had incorrect pitch to the absolute-pitch subjects, and their relative-pitch judgments suffered with such an incorrect reference.

One shortcoming of the present experiment is the absence of a control group of listeners lacking absolute pitch. However, it is implausible that listeners without absolute pitch are able to distinguish between in-tune and deviant references on an absolute basis, and it may be assumed that those listeners perform equally well whether the reference is in tune or deviated from the standard pitch.

\section{EXPERIMENT 2}

The purpose of Experiment 2 was to examine further the relative-pitch performance of absolute-pitch listeners. In Experiment 1, there were a number of intermediate pitches to be judged; in Experiment 2, only the 12 conventional relative-pitch categories within an octave were used. In Experiment 1, the deviant reference tones were only slightly altered from the in-tune $\mathrm{C}$ (a 50-cent deviation at the most). Such slight deviations may have had only a small influence on relative-pitch judgment and/or may possibly have allowed the subjects to do the task solely on the basis of absolute pitch. Experiment 2 included reference tones that were clearly different from $\mathrm{C}$, so that the subjects were forced to do the task on the basis of relative pitch. The pitch of the reference was set at the in-tune $C, F \#$, or the out-of-tune $\mathrm{E}$ (a quarter-tone-lower $\mathrm{E}$ ). It was expected that such a large difference between the reference pitches would produce a more obvious effect.

\section{Method}

Subjects. Eleven undergraduate music students were selected as absolute-pitch subjects (AP group) in the same manner as the sub- 
jects in Experiment 1. All of them had received musical training on the piano from 3 to 6 years of age, and 9 out of 11 were in courses for music education in Niigata University. For comparison, there were an additional 6 subjects who had no absolute pitch (NAP group). They had various musical backgrounds; 2 of them had begun piano lessons from 5 years old, and the others from 7 to 8 years old. Four of the NAP group were in music education courses, and the other 2 were members of an amateur chorus group.

Stimulus and Apparatus. The stimuli consisted of 12 ascending melodic intervals ranging from a semitone to an octave. The first tone of the interval was the reference tone. There were three different references: $C_{4}(261.6 \mathrm{~Hz}), \mathrm{F}_{4}(370.0 \mathrm{~Hz})$, and a quarter-tonelower $\mathrm{E}_{4}(320.2 \mathrm{~Hz})$. The fundamental frequency of the second tone (the target) was determined relative to a given reference so as to form 1 of 12 musical intervals in chromatic scale tuned according to equal temperament. There were thus no mistuned intervals. Each stimulus tone was a synthesized piano-like tone with an amplitude envelope of fast attack and slow decay. Its duration was about $0.9 \mathrm{sec}$, and the time interval between the onset of the two tones was $1.0 \mathrm{sec}$.

The apparatus used was the same as that in Experiment 1 except that the stimuli were generated by a different synthesizer module (Yamaha TX-816).

Procedure. The same procedure as in Experiment 1 was used except that, here, there were only 12 relative-pitch categories within an octave as possible responses. Response time (time interval between the onset of the target tone and the occurrence of the response) was measured as well as response accuracy. Experimental trials with different references were ordered pseudorandomly, with the constraint that the same reference did not appear consecutively. Overall, five trials were given for each stimulus interval and each reference tone.

\section{Results and Discussion}

Figure 3 displays average percentages of correct identification for different intervals in the different reference conditions. Most notably, the AP group (represented by filled symbols) gave markedly different performances depending on reference condition. The performance was at the highest level in the $C$ reference condition, whereas it significantly declined in the below-E and $F \#$ reference conditions. A repeated measures two-way ANOVA on the data of the AP group with reference and interval as withinsubject factors revealed that the main effect of reference was highly significant $[F(2,20)=18.86, p<.001]$. Planned comparisons among means showed that the average percentage correct in the $\mathrm{C}$ reference condition was significantly higher than the combined means of the other two reference conditions $[F(1,20)=18.86, p<.001]$. There was no significant difference between the below-E and $\mathrm{F} \#$ reference conditions $[F(1,20)=.597, p>.4]$.

Furthermore, the identification performance shows a considerable difference among different stimulus intervals; generally, the most accurately identified were certain small intervals, such as minor 2 nd and major 2 nd, and certain important intervals in the diatonic scale, such as the perfect fifth and octave. By contrast, nondiatonic larger intervals, such as the tritone, minor 6 th, minor 7 th, were most poorly identified. The main effect of interval was shown to be highly significant $[F(11,110)=7.19, p<$ $.0001]$. The effect of interval significantly interacted with the effect of reference $[F(22,220)=2.04, p<.05]$. This interaction is seen in the result that the performance decline in the below-E and $\mathrm{F} \#$ reference conditions was more pronounced for the larger nondiatonic intervals, which were least accurately identified, relative to those intervals most accurately identified.

In marked contrast, the NAP group (represented by open symbols) showed no consistent differences in performance among the three reference conditions $[F(2,8)=$ $1.97, p>.2]$. Only the main effect of interval was significant $[F(11,44)=4.41, p<.02]$, reflecting the tendency that performance markedly deteriorated for some larger nondiatonic intervals (such as the tritone and minor 6th). The interaction between reference and interval was not significant $[F(22,88)=1.10, p>.3]$, indicating that the

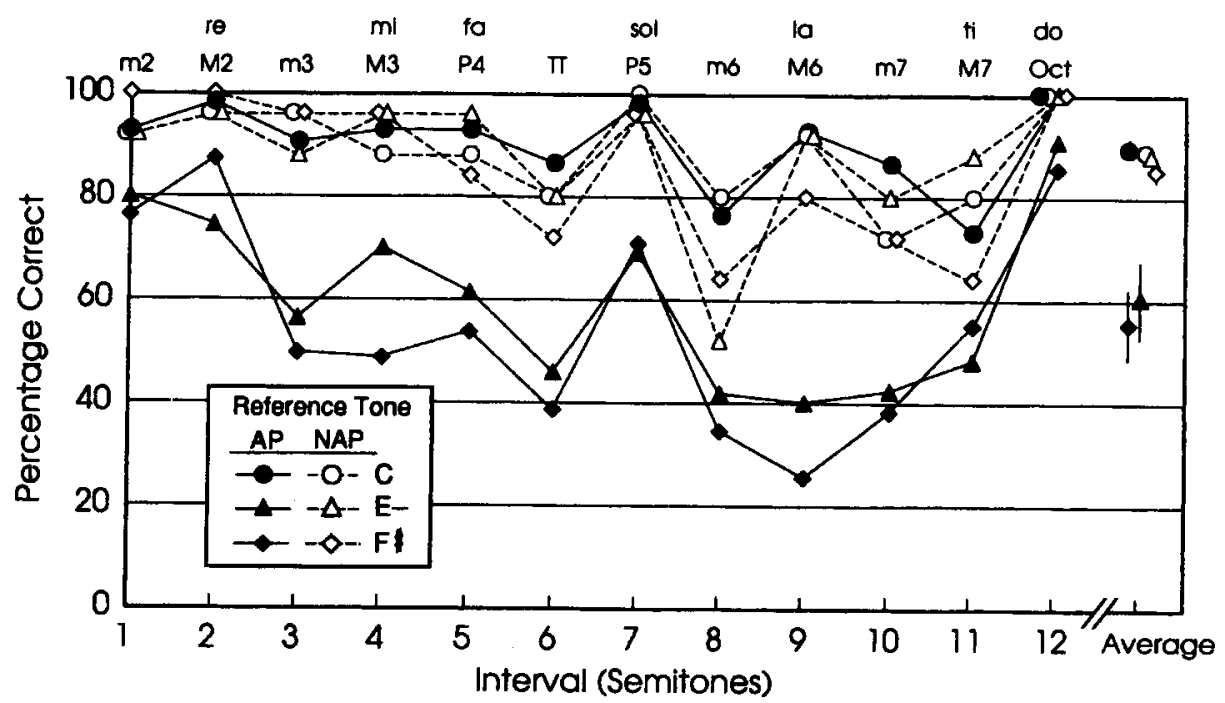

Figure 3. Average percentage of correct identification of musical intervals as a function of musical interval size in different reference conditions. Filled symbols represent data for 11 absolute-pitch listeners, and open symbols represent data for 6 non-absolute-pitch listeners. Error bars on overall average data show $\pm 1 S E$. 
performance as a function of interval showed a similar pattern across three reference conditions.

Figure 4 shows the average response time as a function of interval. There is a coincidence between response speed and accuracy in relative-pitch identification, indicating that the two variables measure the same thing. Pearson's correlation coefficients calculated between the percentage correct and the response time for different intervals were strongly negative in all key conditions $(r=-.868$ to $-.894)$ for the AP group, and moderately negative ( $r=$ -.439 to -.796 ) for the NAP group.

A repeated measures two-way ANOVA on the response time data of the AP group demonstrated a highly significant main effect of reference $[F(2,20)=28.98, p<.0001]$. Planned comparisons among means showed that the average response time in the $\mathrm{C}$ context was significantly faster than the combined means of the other two contexts $[F(1,20)$ $=56.08, p<.001]$, but there was no significant difference between the below-E and $\mathrm{F} \#$ contexts $[F(1,20)=1.88, p>$ .15]. Also significant were a main effect of interval $[F(11,110)=13.49, p<.0001]$ and an interaction of reference and interval $[F(22,220)=2.62, p<.002]$. Generally, response times were different among intervals; intervals most quickly identified were small intervals that might be melodically important and easily perceived (such as the major 2 nd and minor 2 nd) and certain intervals that are important in the diatonic scale (such as the perfect 5 th and octave). In contrast, it took a longer time to identify certain larger intervals, such as the tritone, minor 6 th, major 6 th, minor 7 th. This may have resulted from a combination of two factors: larger intervals are more difficult to perceive, and nondiatonic intervals are less accessible in the tonal hierarchy. The significant interaction of reference and interval indicates that the difference in response time between reference conditions is more striking in the larger or nondiatonic intervals than in the smaller or diatonically important intervals. Note that the octave is an exceptional case in which response time is almost the same in the three context conditions, suggesting that the octave was most easily and quickly identified on the basis of the octave equivalence.

Response time of the NAP group again demonstrated a marked contrast to that of the AP group. There was no significant effect of reference $[F(2,8)=0.124]$, nor was there a significant interaction between reference and interval $[F(22,88)=1.281, p>.2]$; only the effect of interval was significant $[F(11,44)=4.01, p<.001]$. Response time was almost the same regardless of the reference. This is expected from the invariance of musical interval under transposition. The significant difference in response time among the intervals can be interpreted as reflecting the same general tendency in interval identification and the effect of tonal hierarchy as shown for the AP group.

Overall performance and response speed in the two groups is depicted by symbols on the right side of Figures 3 and 4 . In the C condition, the AP and NAP groups were roughly matched in performance level $[t(14.9)=$ $0.55, p>.5]$ and response time $[t(12.6)=1.34, p>.2]$; in contrast, in the other two conditions, the AP group was significantly below the NAP group in performance level $[t(10.6)=3.5, p<.006$, for the below-E context, and $t(11.9)=3.92, p<.003$, for the $\mathrm{F} \#$ context] and significantly slower in response speed than the NAP group $[t(13.6)=3.60, p<.005$, for the below-E context, and $t(12.4)=4.28, p<.002$, for the F\# context].

The error patterns the AP subjects made in the below$\mathrm{E}$ and $\mathrm{F} \#$ conditions are depicted in Figure 5 as confusion matrices. Most errors are located in the vicinity of the diagonal line representing correct responses, suggesting that the AP subjects were generally inaccurate in identifying

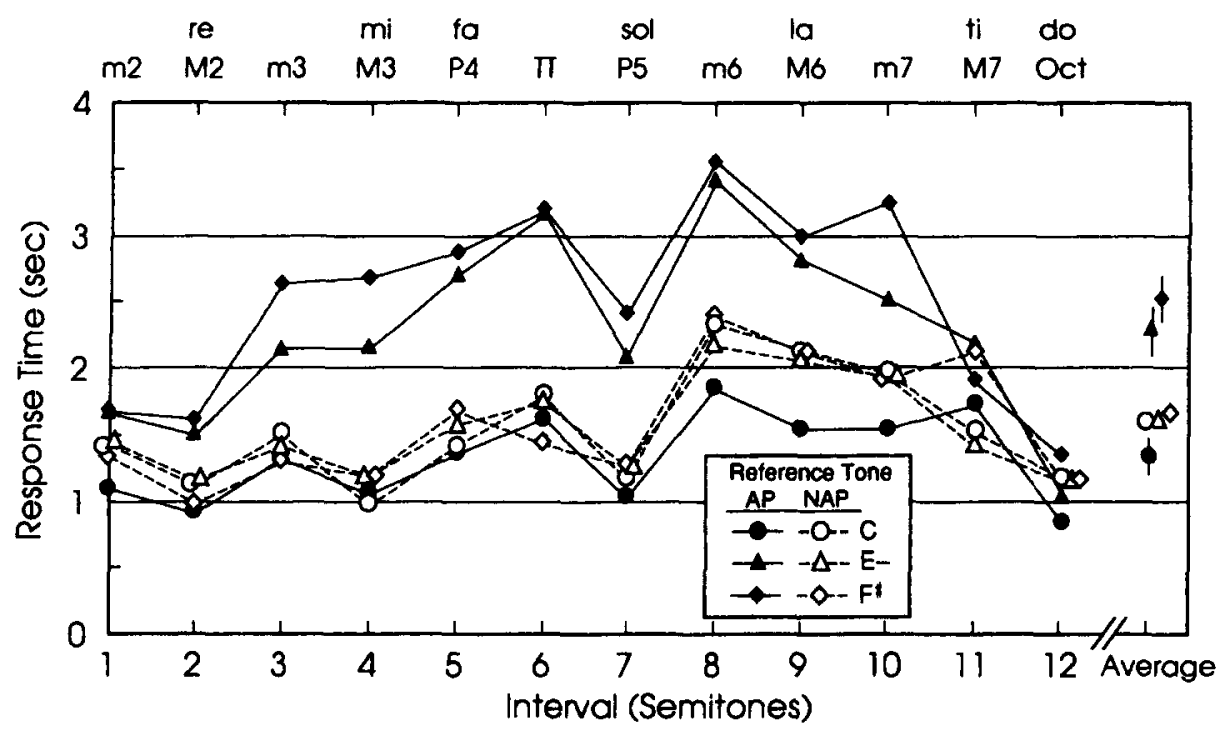

Figure 4. Average response times of identification responses as a function of musical interval size in different reference conditions. Filled symbols represent data for 11 absolute-pitch listeners, and open symbols represent data for 6 non-absolute-pitch listeners. Error bars on overall average data show $\pm 1 S E$. 

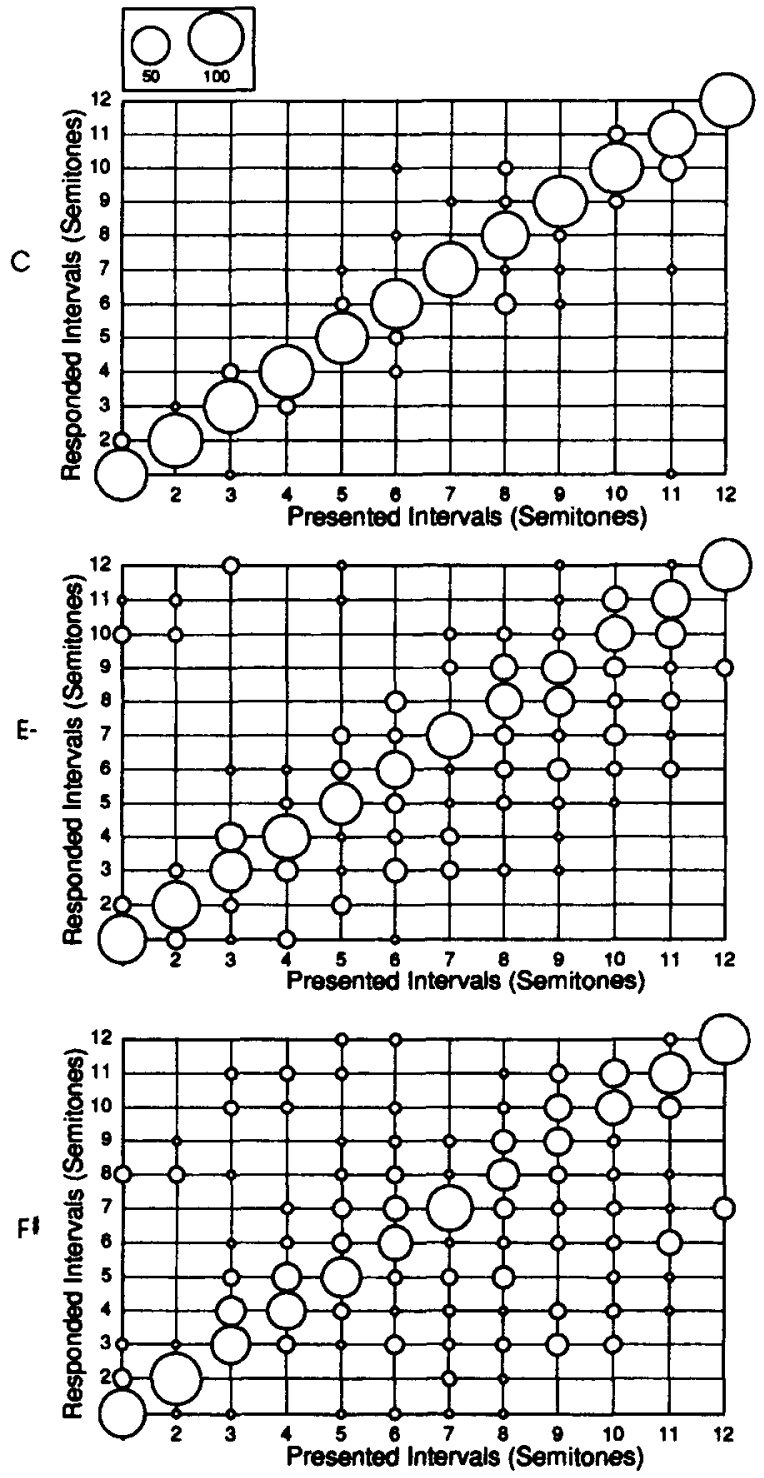

Figure 5. The scatter patterns of responses from the AP group. The upper panel is for the $C$ condition, the middle for the below$E$ condition ( $E-$ ), and the lower panel for the $F \#$ condition. The area of each circle is proportional to the number of responses to corresponding stimulus interval.

intervals in the context of references other than C. Also notably, there was a small number of responses shifted from the correct ones by 3 or 6 semitones, an amount roughly equal to the distance between the below-E or F\# reference and $C$. Errors of this type were typically observed from 3 out of 11 AP subjects. These subjects may have sometimes carelessly named the absolute pitch of the second tone of the interval instead of its relative pitch.

\section{GENERAL DISCUSSION}

The findings obtained from the present experiments extend the finding of the previous report (Miyazaki, 1992). In Experiment 1, 6 out of 8 subjects with absolute pitch showed a decline in performance in relative-pitch identification when the reference tone deviated noticeably from the pitch standard. Experiment 2 provided more convincing evidence on the disadvantageous aspect of absolute pitch in relative-pitch processing. In contrast to the subjects in the NAP group, the subjects in the AP group revealed a marked deterioration of performance and a significant lengthening of response time when the reference was below-E and F\#, as compared with when it was $C$.

Historically, there is a controversy over the notions of fixed-scale anchors or subjective standards for absolute pitch. Cuddy (1968) reported that it was effective to use a single anchor note in training adult listeners without absolute pitch to have long-term retention of certain notes. Following Cuddy's method, Brady (1970) trained himself extensively and achieved a remarkable improvement in absolute-pitch identification on the basis of a fixed-scale mechanism anchored to a particular reference tone. However, Corliss (1973) argued, from his subjective experience as an absolute-pitch possessor, that absolute-pitch listeners really perceive tone chroma specific to each single note without relying on relative pitch with reference to a fixed anchor. For him, simultaneous notes are recognized first as their respective pitch classes and then as a chord. It could be argued that what adult listeners can acquire through training with fixed anchors is not genuine absolute pitch of a set of pitch classes but pitch relations relative to a fixed anchor, corresponding to a sort of quasiabsolute pitch as defined by Bachem (1937). Most listeners in the present experiments are believed to be genuine absolute-pitch possessors who acquired it in early childhood. They should have no need of using the fixed-scale mechanism in identifying pitch classes, and, therefore, the notion of fixed anchors for absolute pitch is untenable for them.

Hypothetically, two different strategies were available to the absolute-pitch subjects in the present experiments. Under the "absolute-pitch strategy," they would at first identify separately the pitch classes of two tones comprising the interval to be identified, then calculate the interval. In this strategy, musical intervals are only indirectly perceived, possibly by counting the number of steps between the two tones demarcating the interval. Another is the "relative-pitch strategy," under which the pitch relation of the target tone to the reference is abstracted directly and independently of the absolute-pitch level.

In ordinary musical situations, absolute-pitch listeners would presumably take advantage of their endowed ability and give an excellent performance in a pitch-naming task. However, Ward and Burns (1982) have pointed out that if absolute-pitch listeners stick to the absolute-pitch strategy and are unable to avoid categorizing individual tones, they would be handicapped in recognizing musical intervals in a particular situation where tones comprising the intervals were slightly out of tune. Specifically, they would categorize both pitches demarcating the interval, and accumulated rounding errors could result in erroneous identification of the interval. For example, when a 30-cent-higher $\mathrm{C}$ and a 30-cent-lower $\mathrm{E}$ are presented, an 
absolute-pitch listener might use the absolute-pitch strategy and categorize these tones as $\mathrm{C}$ and $\mathrm{E}$, respectively, neglecting their slight deviations and name the interval as the major $3 \mathrm{rd}$. By contrast, a subject who used the relativepitch strategy would hear the interval as the minor $3 \mathrm{rd}$ more often than the major $3 \mathrm{rd}$, since the interval was actually 340 cents, which was closer to the minor 3 rd than the major 3rd.

Benguerel and Westdal (1991) attempted to determine which of the strategies absolute-pitch listeners used in identifying musical intervals, by examining their responses to certain critical intervals. They reasoned that if the subjects' responses suggested the effect of accumulated rounding errors as described above, it could be concluded that they used the absolute-pitch strategy. They found no such effect and concluded that the subjects did not apply their absolute-pitch ability to the task of musical interval identification.

A tentative analysis similar to that of Benguerel and Westdal (1991) was carried out on a portion of the present data from Experiment 1. Some critical intervals in the conditions in which the reference tones deviated by 16 and 30 cents from the in-tune $C$ were analyzed; these intervals were expected to be named differently according to which strategies (absolute or relative) the subjects would adopt. The analysis revealed that, for 4 out of 8 absolute-pitch subjects, the obtained results were closer to the prediction based on the relative-pitch strategy than on the absolutepitch strategy; for the other 4 subjects, there were no marked differences in predictability between the assumptions of these two strategies. Apparently, this analysis indicates that most absolute-pitch subjects used mainly relative pitch in Experiment 1.

This analysis is not conclusive, since Experiment 1 was not optimally designed for differentiating between the strategies. If the absolute-pitch listeners could use the relative-pitch strategy perfectly, they could hear a pitch relationship between the reference and the target tones and could have achieved equal performance in any reference condition. Why did they not do so? In Experiment 1, some subjects apparently could not suppress their absolutepitch sense completely and perceived the certain deviant references as out of tune, resulting in the lowered performance in identifying intervals. More convincingly, in Experiment 2 , the absolute-pitch listeners made more errors and took a longer time in identifying musical intervals than did the listeners without absolute pitch in the non-C reference conditions. They may have sometimes relied on absolute pitch in performing the task. The absolute-pitch strategy worked quite well in the $\mathrm{C}$ reference condition, because naming musical intervals with the $\mathrm{C}$ reference was a well-practiced skill. It is possible, however, that they abandoned that strategy in the non- $\mathrm{C}$ reference conditions because this strategy did not work with the non- $\mathrm{C}$ references, and instead they attempted the relative-pitch processing, which was poorly practiced. According to this hypothesis, these listeners are weak in relative-pitch processing due to a lack of training in this skill. On the other hand, some absolute-pitch listeners never attempted to perceive musical intervals as such but stuck to the absolute-pitch strategy in every reference condition. In fact, some listeners reported that, in order to identify the musical interval name, they tried to count the number of tonal steps between the presented tones on their fingers or on an imaginary keyboard.

The lowered performance the absolute-pitch listeners showed in the non- $\mathrm{C}$ reference conditions as opposed to the $\mathrm{C}$ condition can be accounted for not only by the strategies they used but also by their inappropriate manner in naming relative pitch. They were required to identify the musical pitch relations using either the musical interval names or the sol-fa names (e.g., mi flat, mi, fa, etc.) as labels. In effect, most subjects used the sol-fa naming system, because it was much more familiar than was the interval naming system. The listeners lacking absolute pitch presumably used the sol-fa labels as relative-pitch names based on the movable-do system, but the absolutepitch listeners seemed to use the sol-fa labels as absolutepitch names in the fixed-do manner. In fact, most of the absolute-pitch subjects reported that they had developed a tendency to use the sol-fa names as substitutes for absolute-pitch names, fixedly naming $\mathrm{C}$ as do. If they responded in this manner, it would be quite easy for them to identify the target tone relative to the $C$ (do) reference, since all they had to do was to answer the sol-fa name of that tone. Although this advantage would disappear when the reference was not $\mathrm{C}$, it is likely that, in such cases, they would stick to the absolute-pitch strategy associated with the usage of the sol-fa names as absolute-pitch labels and would take great pains to transpose the pitch interval between the non- $\mathrm{C}$ reference and the target to a more familiar position with a fixed $\mathrm{C}$ reference. Furthermore, even if they could perceive the pitch relationships, they should have difficulty in using musical interval or relative-pitch labels.

The observed marked decline in performance of the $a b-$ solute-pitch subjects seen in the F\# and below-E reference conditions of Experiment 2 could be explained partially by such possible efforts and the difficulty they had in naming relative pitches. If the listeners are skilled in relative pitch, they should have a firmly established naming system for relative pitches. Therefore, the observation that most absolute-pitch listeners had not firmly established the relative-pitch naming system may be, in itself, regarded as additional evidence indicating that they cannot free themselves from absolute pitch and are relatively weak in relative-pitch processing.

The present experiments may have an implication for experimental psychologists who are engaged in perception and memory of musical pitch. It has been demonstrated that some absolute-pitch listeners have a tendency to rely on absolute-pitch sense when processing musical pitch relations and are relatively weak in perceiving musical pitches in a tonal context. As a consequence, it is probable that they give an excellent score in certain pitch tasks in which absolute pitch is useful. In contrast, as demonstrated in the present experiments, they may show lower performance in some other tasks in which relative- 
pitch processing is required and absolute pitch is of no use. There are various types of absolute pitch differing in accuracy (Miyazaki, 1990), and there are different types of strategies used by absolute-pitch listeners in different musical pitch-processing tasks, depending on an individual's musical history. Moreover, the proportion of absolute-pitch possessors among musically experienced people is higher than believed earlier (Miyazaki, 1988), and there may even be absolute-pitch possessors without self-awareness or with latent absolute pitch. Experimental psychologists in these areas should therefore take every possible care to ascertain possible possession of absolute pitch by their subjects. It may be possible that such subjects could influence the outcome in experiments by using various kinds of absolute-pitch strategy. Further research is needed to examine how absolute-pitch listeners process musical pitch in more musically sophisticated situations where tonal melodies, harmonic sequences, and key relationships are involved.

\section{REFERENCES}

Attneave, F., \& Olson, R. K. (1971). Pitch as medium: A new approach to psychophysical scaling. American Journal of Psychology, 84, 147-166.

BACHEM, A. (1937). Various types of absolute pitch. Journal of the Acoustical Society of America, 9, 146-151.

Benguerel, A. P., \& Westdal, C. (1991). Absolute pitch and perception of sequential musical intervals. Music Perception, 9, 105-120.

Brady, P. T. (1970). Fixed-scale mechanism of absolute pitch. Journal of the Acoustical Society of America, 48, 883-887.

Burns, E. M., \& WARD, W. D. (1978). Categorical perception-Phenomenon or epiphenomenon: Evidence from experiments in the perception of melodic musical intervals. Journal of the Acoustical Society of America, 63, 456-468.

CorLiss, E. L. (1973). Remark on "Fixed-scale mechanism of absolute pitch." Journal of the Acoustical Society of America, 53, 1737-1739.

Cuddy, L. L. (1968). Practice effects in the absolute judgment of pitch. Journal of the Acoustical Society of America, 43, 1069-1076.

Hulse, S. H. (1989). Comparative psychology and pitch pattern per- ception in songbirds. In R. J. Dooling \& S. H. Hulse (Eds.), The comparative psychology of audition: Perceiving complex sounds (pp. 331349). Hillsdale, NJ: Erlbaum

Hulse, S. H., TAKeuChI, A. H., \& BRAaten, R. F. (1992). Perceptual invariances in the comparative psychology of music. Music Perception, 10, 151-184.

Krumhansl, C. L. (1990). Cognitive foundations of musical pitch. New York: Oxford University Press.

Krumhansl, C. L., \& Kessler, E. J. (1982). Tracing the dynamic changes in perceived tonal organization in a spatial representation of musical keys. Psychological Review, 89, 334-368.

KRUMHANSL, C. L., \& SHEPARD. R. N. (1979). Quantification of the hierarchy of tonal functions within a diatonic context. Journal of Experimental Psychology: Human Perception \& Performance, 5, 579-594.

Lerdahl, F. (1988). Tonal pitch space. Music Perception, 5, 315-350.

MiYAZAKI, K. (1988). The identification of musical pitch by absolute pitch possessors. Perception \& Psychophysics, 44, 501-512.

MIYAZAKI, K. (1989). Absolute pitch identification: Effects of timbre and pitch region. Music Perception, 7, 1-14.

MiYAZAKI, K. (1990). The speed of musical pitch identification by absolute-pitch possessors. Music Perception, 8, 177-188.

MIYAZAKI, K. (1992). Perception of musical intervals by absolute pitch possessors. Music Perception, 9, 413-426.

Oura, Y., \& EGuchI, K. (1982). Absolute pitch training program for children. Ongaku Kyouiku Kenkyu (Music Education Research), 32, 162-171. (in Japanese)

Piston, W. (1987). Harmony (5th ed.). New York: Norton.

Rakowski, A. (1990). Intonation variants of musical intervals in isolation and in musical contexts. Psychology of Music, 18, 60-72.

Sergeanr, D., \& Roche, S. (1973). Perceptual shifts in the auditory information processing of young children. Psychology of Music, 1, 39 . 48.

Siegel, J. A., \& Siegel, W. (1977). Absolute identification of notes and intervals by musicians. Perception \& Psychophisics, 21, 143-152.

Takeuchi, A. H., \& Hulse, S. H. (1991). Absolute-pitch judgments of black- and white-key pitches. Music Perception, 9, 27-46.

Takeuchl, A. H., \& Hulse, S. H. (1993). Absolute pitch. Psuchological Bulletin, 113, 345-361.

Ward, W. D., \& Burns, E. M. (1982). Absolute pitch. In D. Deutsch (Ed.), The psychology of music (pp. 431-451). New York: Academic Press.

(Manuscript received November 9, 1993; revision accepted for publication January 15, 1995.) 